\title{
Type-Specific Screen Time Associations with Cardiovascular Risk Markers in Children
}

\author{
Emmanuel Stamatakis, PhD, Ngaire Coombs, PhD, Russell Jago, PhD, Augusta Gama, PhD, \\ Isabel Mourão, PhD, Helena Nogueira, PhD, Vítor Rosado, PhD, Cristina Padez, PhD
}

\begin{abstract}
Background: There is evidence that TV time may have stronger associations with cardiovascular risk markers than other types of screen time, but most studies focus on TV, or total screen time.

Purpose: To examine associations between types of screen time and cardiovascular risk markers in school-age children.

Methods: Cross-sectional study of 2515 school children in Portugal (aged 2-12 years, data collected in 2009 and 2010). Three types of screen time (TV, electronic games, and PC time) were collected through a questionnaire, and data on three cardiovascular risk markers (resting heart rate; diastolic blood pressure [DBP]; and systolic blood pressure [SBP]) were collected by a trained fieldworker. Complex-samples generalized linear models were run for each combination of screen time predictor and cardiovascular risk outcome, and a clustered cardiovascular risk score, adjusting for potential confounders (including physical activity). Analyses were conducted in 2011 and 2012.
\end{abstract}

Results: TV viewing, but not PC or electronic games time, was associated positively with clustered cardiovascular risk score, DBP, and SBP after adjustment for all covariates. Watching TV for 2 hours/day (compared to 1 hour/day) was associated with higher DBP (coefficient, logged and back-transformed $0.02,95 \%$ CI $\quad 0.00,0.04$, linear trend $p \quad 0.003$ ); SBP (logged and back-transformed $0.02,95 \% \mathrm{CI} \quad 0.01,0.05, p \quad 0.009)$, and clustered cardiovascular risk score $(0.13,95 \%$ CI $0.02,0.24, p \quad 0.001)$.

Conclusions: TV viewing, but no other type of screen time, was associated positively with cardiovascular risk markers independently of physical activity. Studies using a single marker of screen time or sedentary behavior may conceal screen time type-specific associations.

\section{Introduction}

$\mathrm{P}$ hysical activity is important for children's cardiovascular health. ${ }^{1-3}$ Recent evidence suggests that the time children spend being sedentary (as characterized by activities involving sitting) also may be asso-

ciated with cardiovascular health, independently of time

From the Department of Epidemiology and Public Health (Stamatakis, Coombs), and the Physical Activity Research Group (Stamatakis, Coombs), University College London, London; the Centre for Exercise, Nutrition and Health Sciences (Jago), School for Policy Studies, University of Bristol, Bristol, United Kingdom; the Faculty of Sciences (Gama), University of Lisbon; the Tropical Research Institute (Rosado), Lisbon; the Centre for Anthropology and Health (Gama, Rosado, Padez), the Department of Geography (Nogueira), and the Department of Life Sciences (Padez), University of Coimbra, Coimbra; and the Research Center in Health and Human Development (Mourão), University of Tras-os-Montes and Alto Douro, Vila Real, Portugal

Address correspondence to: Emmanuel Stamatakis, PhD, Department of Epidemiology and Public Health, University College London, 1-19 Torrington Place, London WC1E6BT, UK.E-mail: e.stamatakis@ucl.ac.uk. spent being physically active. ${ }^{4,5}$ Screen time (e.g., TV viewing, playing electronic games [games], and time spent at a personal computer [PC]) is one of the largest components of sedentary behavior. National clinical and public health recommendations for physical activity have included screen time, with the most common recom-

mendation being that TV time be limited to 2 hours per day or less for children and adolescents. ${ }^{6,9}$

More than $45 \%$ and $47 \%$ of boys and girls in England aged $2-15$ years spent more than 2 hours a day watching TV, and even more on weekends. ${ }^{10}$ TV viewing may have stronger associations with cardiovascular risk indicators than other types of sedentary behavior for both children ${ }^{4}$ and adults. ${ }^{11}$ Evidence also indicates that TV viewing has stronger associations with cardiovascular risk indicators in children than does total sedentary behavior, as measured by accelerometers. ${ }^{12,13}$ However, associations are not consistent, and some studies focus on either TV viewing alone $\mathrm{e}^{3,14}$ or an aggregate measure of screen time, ${ }^{5,15}$ potentially missing type-specific associations between screen time and cardiovascular risk 
markers. Identifying type-specific associations between screen time and cardiovascular risk markers is important for the refinement of public health recommendations and the design of behavioral interventions aimed at reducing screen time in children.

The aim of the present study was to examine the differential associations of three types of screen time with cardiovascular risk indicators in a large population sample of schoolchildren from Portugal. To the authors' knowledge, this is the first study to examine the associations between multiple indicators of screen time and cardiovascular risk in children living in Southern Europe.

\section{Methods}

\section{Sample and Sampling Design}

Data used in the current study were drawn from the Portuguese Prevalence Study of Obesity in Childhood (PPSOC), a crosssectional study conducted between March 2009 and January 2010 in mainland schools in Portugal. Details on sampling and response rates can be found elsewhere. ${ }^{16}$ Briefly, private and public schools were selected randomly from the Ministry of Education database. Sampling was based on a gender- and age-specific proportionate stratified random design with district as the primary sampling unit. Overall parental consent was $57.4 \%$ (100\% for those included in the final analysis). A total of 17,509 children aged 2-13 years were recruited, among whom 2515 children aged 2-12 years also had clinical measurements and were included in the present analyses. Ethical approval for PPSOC was given by Direcção Geral de Inovação e Desenvolvimento.

\section{Measurements}

Screen time was assessed by proxy parental questionnaires, which were provided to consenting parents by the school. Parents were asked to report the average number of hours per day that the child spent watching TV on a weekday, a Saturday, and a Sunday. Response options for all questions were none, 1 hour, 1 hour, 2 hours, 3 hours, 4 hours, and 5 hours. Parents also were asked to report child PC and game use on a weekday, a Saturday, and a Sunday. PC and games use questions had the same response options as the TV-viewing items above. The questionnaires collected information on physical activity, including physical activity at school (hours/week); mode and duration of travel to/from school (walking or cycling were considered to be physical activity); and minutes per week spent playing sports outside of school.

Trained research workers measured diastolic (DBP) and systolic (SBP) blood pressure ( $\mathrm{mmHg}$ ); resting heart rate (RHR, in beats/ minute); skinfold thickness (mm; triceps, subscapular, and suprailiac); height ( $\mathrm{m})$; and weight ( $\mathrm{kg}$ ). Height was measured using a Seca 217 stadiometer; weight using Seca 770 scales; skinfolds using a Holtain Ltd. skinfolds caliper; and DBP, SBP, and RHR using an Omron M7 blood pressure monitor. Three measurements were taken each for DBP, SBP, and RHR, and the means of the three measurements were used in analyses.
Other information collected in the questionnaire included age and contextual factors (frequency of consumption of certain key foods such as sodas, iced tea, cakes, chocolate, hamburgers and pizza; 
number of fruit portions eaten per week; parental BMI; number of hours of sleep per night; birth weight; and breastfeeding duration). SES indicators also were included (parental education, parental employment status, perception of crime in neighborhood).

\section{Data Handling}

A clustered cardiovascular risk score was calculated from the five risk markers: RHR; DBP; SBP; BMI (calculated by standard formula); and sum of skinfolds, in a process similar to that conducted previously. ${ }^{3,17,18}$ After log-transformation of SBP, DBP, and sum of skinfolds, and $z$-score conversion $[z \quad($ value $-\mathrm{M}) / \mathrm{SD}]$ of all cardiovascular variables (average blood pressure was used as an index for SBP and DBP), the $z$-scores were summed, and the sum was divided by 4 to compile the cardiovascular risk score with units of SD.

Sedentary behavior variables (TV, game, and PC time) were recorded separately for weekdays, Saturdays, and Sundays. To obtain an average daily value for TV, PC, and game time, the original categories $(0,1,1,2,3,4,5$ hours $)$ were recoded into a continuous variable: none was coded as $0 ; 1$ hour as $0.5 ; 1$ hour as $1 ; 2$ hours as 2; and so on. Five hours or more was coded as 5 . These proxy continuous variables were then weighted such that weekdays accounted for five sevenths, and Saturdays and Sundays each accounted for one seventh of the variable.

For the analyses, the continuous average daily TV-viewing time variable was recategorized into three categories: 1 hour/ day, 1-2 hours/day, and 2 hours/day. Because of lower perday volumes than TV viewing and the resulting low numbers of observations in the higher groups, different cut-offs were used for PC and game time ( 0.5 hours/day, 0.5-1 hour/day, 1 hour/day). The three physical activity variables (physical activity at school, time spent walking or cycling to and from school, and time spent playing sports outside of school) were converted into the same units (minutes per week) and summed into one total physical activity variable. An unhealthy diet score was calculated by summing the frequency of consumption of hamburgers, pizza, sodas, iced tea, cakes, and chocolate, with all groupings weighted equally (weekly range 6-36).

Because of a substantial proportion of cases with at least one missing value in at least one covariable or exposure variable ( $40 \%$ ), multiple imputation was performed. No exposure variable had more than $15.9 \%$ of values missing, and most had a considerably lower percentage. Multiple imputation was performed using SPSS, version 18, with linear regression as the type of imputation and five imputations. Outcome variables were not involved in the imputation process. Main results are presented using the pooled outcomes of five imputed data sets; key analyses using the original data set with listwise deletion are included in Appendixes A-D (available online at www.ajpmonline.org; Appendix A provides more detail on the multiple imputation process).

\section{Data Analysis}

Analyses were conducted in 2011 and 2012. The association between each screen time variable and each of the four cardiovascular risk indicators (DBP, SBP, RHR, and clustered cardiovascular risk score) was examined using generalized linear models and multiple linear regression to determine the trend $p$-value. BMI and sum of skinfolds were not considered as stand-alone outcomes in the present study because a separate paper examines their associations 
with screen time in the full PPSOC data set of 17,000 children (unpublished observations, 2012).

Outliers outside 4 SDs of the mean for any outcome or component of clustered cardiovascular score (including BMI and sum of skinfolds) were removed from the analyses, leaving 2462 cases. DBP, SBP, and sum of skinfolds were logged to further improve normality. Residual statistics and plots for each model were checked for normality, independence of observations, homoscedasticity, influential outliers, and linear relationship between dependent and (continuous) independent variables. The complexsamples generalized linear models procedure was used to produce results with robust SEs that take into account clustering of participants with schools.

Models were adjusted for (1) age and gender; (2) additionally for SES (mother's and father's education) and all other contextual covariables (sleep time, birth weight, duration of breastfeeding, unhealthy diet score, weekly fruit consumption, perception of crime locally, mother's and father's BMI); and (3) additionally for weekly physical activity time and mutually adjusted for TV, game, and PC time. Coefficients from the procedure indicate mean differences (in values for each cardiovascular indicator) between the reference category and each of the other screen-viewing categories. The lowest category ( 1 hour/day for TV and total screen time, 0.5 hours/day for PC and game time) is the reference category for the mean difference in the outcome (and associated CI for the difference) in all complex-samples generalized linear models (i.e., the presented coefficients compare the middle category with the lowest category, and the highest category with the lowest category). All statistical models were run for each combination of outcome (RHR, DBP, SBP, clustered cardiovascular risk score) and predictor (TV, game, and PC time).

There was little evidence of gender or age interactions, so analyses were gender- and age-adjusted but not stratified. Similarly, all main analyses were age-adjusted but not stratified. Because of the broad age range of the sample, and although no evidence was found of age interactions, age-stratified key results (using the median age of 7.1 years to form two age strata) are included in Appendixes A-D (available online at www.ajpmonline.org).

Table 1. Sample characteristics by level of TV viewing ${ }^{a}$

\begin{tabular}{|c|c|c|c|c|c|c|c|}
\hline \multirow[b]{2}{*}{ Categoric variables, ${ }^{\text {b }} \%(n)$} & \multicolumn{5}{|c|}{ Average TV-viewing time per day (hours) } & & \multirow[b]{2}{*}{$p$-value } \\
\hline & 1 & & $1-2$ & & 2 & & \\
\hline Gender (\% male) & $48.5(431)$ & & $52.0(419)$ & & $47.2(238)$ & & 0.186 \\
\hline Father's education (\% 9 years) & $86.1(738)$ & & $83.0(632)$ & & $70.6(329)$ & & $0.001 *$ \\
\hline Mother's education (\% 9 years) & $92.2(811)$ & & 89.4 (712) & & $82.7(402)$ & & $0.001 *$ \\
\hline Area crime (\% strongly disagree unsafe) & $74.0(615)$ & & $72.2(541)$ & & $64.6(299)$ & & $0.001 *$ \\
\hline Continuous variables, ${ }^{\mathrm{c}} \mathrm{M}$ (SD) & & $n$ & & $n$ & & $n$ & \\
\hline Age (years) & $6.98(2.01)$ & 888 & $7.29(1.96)$ & 806 & $7.48(1.95)$ & 504 & $0.001 *$ \\
\hline Resting heart rate (beats/minute) & $89.48(12.46)$ & 888 & $88.44(11.72)$ & 806 & $90.15(12.90)$ & 504 & $0.040 *$ \\
\hline Diastolic blood pressure ( $\mathrm{mmHg}$ ) & $91.67(11.42)$ & 888 & $92.80(11.64)$ & 806 & $95.67(11.62)$ & 504 & $0.001 *$ \\
\hline Systolic blood pressure (mmHg) & $57.13(8.30)$ & 888 & $57.71(8.81)$ & 806 & $59.37(8.84)$ & 504 & $0.001 *$ \\
\hline Cardiovascular risk score & $0.09(0.59)$ & 888 & $0.01(0.64)$ & 806 & $0.16(0.72)$ & 504 & $0.001 *$ \\
\hline $\begin{array}{l}\text { Active travel time to/from school (minutes/ } \\
\text { week) }\end{array}$ & $12.09(35.50)$ & 844 & $15.31(42.15)$ & 764 & $25.54(51.51)$ & 475 & $0.001^{*}$ \\
\hline School physical activity time (minutes/week) & $68.69(29.11)$ & 784 & $73.26(29.18)$ & 727 & $77.96(27.86)$ & 449 & $0.001 *$ \\
\hline Time spent playing sport (minutes/week) & $87.09(104.90)$ & 876 & $79.15(96.20)$ & 796 & $58.42(87.53)$ & 491 & $0.001 *$ \\
\hline Mother's BMI & $23.33(3.56)$ & 840 & $23.70(3.83)$ & 760 & $24.35(4.10)$ & 467 & $0.001 *$ \\
\hline Father's BMI & $26.28(3.29)$ & 793 & $26.23(3.26)$ & 698 & $26.66(3.50)$ & 423 & 0.087 \\
\hline Breastfeeding duration (months) & $4.18(2.92)$ & 784 & $4.15(3.50)$ & 699 & $4.13(3.26)$ & 417 & 0.969 \\
\hline Birth weight (kg) & $3.21(0.53)$ & 848 & $3.22(0.51)$ & 778 & $3.25(0.55)$ & 487 & 0.358 \\
\hline Unhealthy diet score & $15.09(3.14)$ & 807 & $15.63(2.97)$ & 705 & $16.50(3.95)$ & 443 & $0.001 *$ \\
\hline Portions of fruit per day & $2.15(1.30)$ & 862 & $2.05(1.22)$ & 783 & $2.04(1.34)$ & 487 & 0.151 \\
\hline
\end{tabular}

Note: Boldface indicates significance. 
aPrior to multiple imputation, with casewise deletion of missing values

${ }^{b} \mathrm{Chi}$-square test was used to test significance of association between categoric variables and TV viewing ${ }^{\mathrm{C}}$ ANOVA was used to test significance of association between continuous variables and TV viewing.

*p 0.05 
Table 2. Multivariable-adjusted associations between TV viewing frequency and cardiovascular risk markers in Portuguese schoolchildren (n 2462), coefficient $(95 \% \mathrm{Cl})$

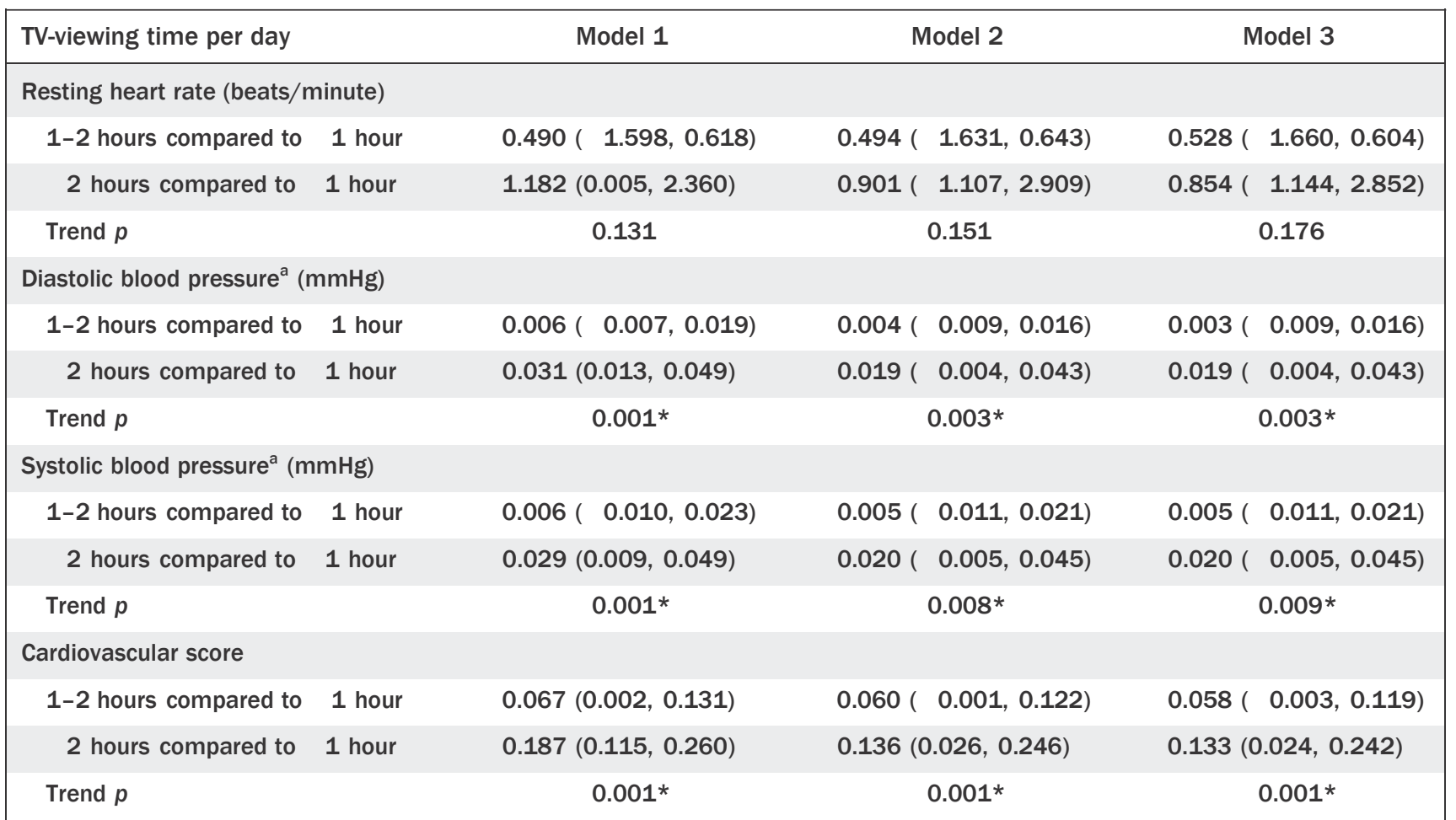

Note: Boldface indicates significance. Model 1 was adjusted for age and gender. Model 2 was further adjusted for mother's education and BMI; father's education and BMI; perception of crime in local area; average hours of sleep per night; birth weight; duration of breastfeeding; bad diet score (based on consumption of sodas, iced tea, cakes, chocolate, hamburgers, and pizza); and number of fruit portions eaten per week. Model 3 was further adjusted for time spent in physical activity per week. Coefficients are generalized linear model coefficients; coefficients indicate mean differences (in cardiovascular risk markers) between the reference category ( 1 hour per day) and each of the other TV-viewing groups (e.g., a value of 0.06 indicates that a specific category had a mean score that is 0.06 units higher than that of the referent group).

${ }^{a}$ Diastolic and systolic blood pressure are logged variables (base 10). Coefficients presented here have been back-transformed to their natural scale by raising 10 to the power of the logged coefficient and deducting 1 .

*p 0.05

\section{Results}

\section{Sample Characteristics}

Table 1 presents the sample characteristics by level of TV viewing (prior to multiple imputation and exclusion of outliers) with casewise deletion of missing values; Appendix B (available online at www.ajpmonline.org) compares the part of the sample that had no missing values in any variable and those who had at least one imputed variable. Participants who watched 2 hours of TV per day were more likely to be older, have parents with 9 years of education, have parents who consider the local area unsafe due to crime, and have a mother with a higher BMI than participants who watched 2 hours per day. They also were more likely to have a higher RHR, DBP, SBP, and a higher clustered cardiovascular risk score. They were more likely to consume unhealthy foods more frequently, and to spend more time on active travel to/from school and on school physical activity while at school, but spend less time on sports outside of school. 


\section{Screen Time indicators and Cardiovascular \\ Risk}

Table 2 shows the results from the models with TV as the main exposure. There was evidence of an association between average daily TV-viewing time and increased DBP, SBP, and clustered cardiovascular risk score, but not RHR. Tables 3 and 4 show the results from the analyses with game time and PC time as the main exposure, respectively. No evidence for an association between game time or PC time and any of the examined outcome variables was found. Fewer associations were observed for the non-imputed, listwise deleted data set (Appendix $\mathrm{C}$, available online at www.ajpmonline.org). PC time and game time were not associated with any cardiovascular outcomes, and TV time was associated only with cardiovascular score.

\section{Age-Stratified Analysis}

Results of the age-stratified analysis were largely in agreement with the age-adjusted results (Appendix D, available online at ). No associations were ob- 
Table 3. Multivariable-adjusted associations between electronic games frequency and cardiovascular risk markers in Portuguese schoolchildren (n 2462), coefficient $(95 \% \mathrm{Cl})$

\begin{tabular}{|c|c|c|c|}
\hline Electronic game time per day & Model 1 & Model 2 & Model 3 \\
\hline \multicolumn{4}{|l|}{ Resting heart rate (beats/minute) } \\
\hline $0.5-1$ hour compared to 0.5 hours & $0.412(0.644,1.468)$ & $0.301(\quad 0.767,1.370)$ & $0.333(0.738,1.403)$ \\
\hline 1 hour compared to 0.5 hours & $0.720(1.786,3.227)$ & 0.458 ( $1.640,2.556)$ & $0.563(1.531,2.657)$ \\
\hline Trend $p$ & 0.421 & 0.528 & 0.470 \\
\hline \multicolumn{4}{|l|}{ Diastolic blood pressure $(\mathrm{mmHg})^{a}$} \\
\hline $0.5-1$ hour compared to 0.5 hours & $0.000(0.011,0.011)$ & $0.002(0.013,0.008)$ & $0.002(0.013,0.009)$ \\
\hline 1 hour compared to 0.5 hours & $0.003(0.023,0.029)$ & $0.004(0.027,0.018)$ & $0.004(0.026,0.019)$ \\
\hline Trend $p$ & 0.891 & 0.655 & 0.691 \\
\hline \multicolumn{4}{|l|}{ Systolic blood pressure $(\mathrm{mmHg})^{a}$} \\
\hline $0.5-1$ hour compared to 0.5 hours & $0.000(0.015,0.015)$ & $0.003(0.017,0.011)$ & $0.003(0.017,0.011)$ \\
\hline 1 hour compared to 0.5 hours & $0.027(0.010,0.066)$ & $0.017(0.021,0.057)$ & $0.018(0.021,0.057)$ \\
\hline Trend $p$ & 0.383 & 0.609 & 0.587 \\
\hline \multicolumn{4}{|l|}{ Cardiovascular score } \\
\hline $0.5-1$ hour compared to 0.5 hours & $0.024(0.040,0.088)$ & $0.007(0.058,0.072)$ & $0.009(0.056,0.075)$ \\
\hline 1 hour compared to 0.5 hours & $0.054(0.109,0.217)$ & $0.009(0.497,0.514)$ & $0.014(0.121,0.149)$ \\
\hline Trend $p$ & 0.354 & 0.771 & 0.682 \\
\hline
\end{tabular}

Note: Boldface indicates significance. Model 1 was adjusted for age and gender. Model 2 was further adjusted for mother's education and BMI; father's education and BMI; perception of crime in local area; average hours of sleep per night; birth weight; duration of breastfeeding; bad diet score (based on consumption of sodas, iced tea, cakes, chocolate, hamburgers, and pizza); and number of fruit portions eaten per week. Model 3 was further adjusted for time spent in physical activity per week. Generalized linear model coefficients; coefficients indicate mean differences (in cardiovascular risk markers) between the reference category ( 1 hour a day) and each of the other TV-viewing groups (e.g., a value of 0.06 indicates that a specific category had a mean score that is 0.06 units higher than the referent group).

${ }^{a}$ Diastolic and systolic blood pressure are logged variables (base 10). Coefficients presented here have been back-transformed to their natural scale by raising 10 to the power of the logged coefficient and deducting 1.

served between PC time or game time and any cardiovascular marker, for either age group. TV time was associated with all cardiovascular outcomes for both age groups, with the exception of SBP in the older age group (7.1-12.3 years) and RHR in the younger age group (2.8-7.1 years).

\section{Discussion}

The present study uses unique data on a large population sample of schoolchildren living in Portugal to examine the differential associations of three screen-time indicators with cardiovascular risk markers. TV-viewing time, but not PC time or electronic game time, showed consistent positive associations with most outcomes. A direct research implication of these results is that the type of screen-time indicator is important when looking at associations with health markers. An indirect implication, which needs to be verified by prospective and interventional research, is that interventions designed to reduce sedentary time may need to focus on TV viewing rather than other forms of screen time. 
Previous studies examining the association between TV viewing and cardiovascular risk markers that took into account physical activity have reported results concordant with those reported here. ${ }^{4,5,12,13}$ Other studies ${ }^{4,19}$ also have found non-TV types of sedentary behavior to be unrelated to cardiovascular risk, once physical activity and other potential confounders are taken into account. In contrast, in a study of 1921 children aged 9-10 years or 15-16 years from three regions in Europe, Ekelund et al. ${ }^{3}$ found no association between TV viewing and clustered cardiovascular risk (derived from body fatness, blood pressure, fasting triglycerides, high-density lipoprotein cholesterol, glucose and insulin levels), after controlling for physical activity and other confounders.

Other studies also have found that associations between sedentary behavior and cardiovascular risk vary by the type of cardiovascular risk indicator. Danielsen et al. ${ }^{5}$ did not find associations between TV viewing and total or high-density lipoprotein (HDL) cholesterol in a sample of 43 children aged $7-13$ years in Norway. However, they 
Table 4. Multivariable-adjusted associations between PC frequency and cardiovascular risk markers in Portuguese schoolchildren ( $n$ 2462), coefficient $(95 \% \mathrm{Cl})$

\begin{tabular}{|c|c|c|c|}
\hline PC time per day & Model 1 & Model 2 & Model 3 \\
\hline \multicolumn{4}{|l|}{ Resting heart rate (beats/minute) } \\
\hline $0.5-1$ hour compared to 0.5 hours & $0.396(0.826,1.617)$ & $0.271(0.963,1.505)$ & $0.304(0.922,1.531)$ \\
\hline 1 hour compared to 0.5 hours & $0.997(0.693,2.687)$ & $0.808(0.930,2.546)$ & $0.779(0.982,2.541)$ \\
\hline Trend $p$ & 0.310 & 0.390 & 0.389 \\
\hline \multicolumn{4}{|l|}{ Diastolic blood pressure ${ }^{a}(\mathrm{mmHg})$} \\
\hline $0.5-1$ hour compared to 0.5 hours & $0.005(0.019,0.009)$ & $0.006(0.020,0.009)$ & $0.005(0.019,0.009)$ \\
\hline 1 hour compared to 0.5 hours & $0.003(0.021,0.014)$ & $0.009(0.026,0.008)$ & $0.009(0.026,0.008)$ \\
\hline Trend $p$ & 0.498 & 0.264 & 0.210 \\
\hline \multicolumn{4}{|l|}{ Systolic blood pressure ${ }^{a}(\mathrm{mmHg})$} \\
\hline $0.5-1$ hour compared to 0.5 hours & $0.005(0.019,0.010)$ & $0.006(0.022,0.011)$ & $0.006(0.022,0.011)$ \\
\hline 1 hour compared to 0.5 hours & $0.006(0.026,0.014)$ & $0.011(0.031,0.010)$ & $0.011(0.032,0.010)$ \\
\hline Trend $p$ & 0.512 & 0.313 & 0.314 \\
\hline \multicolumn{4}{|l|}{ Cardiovascular score } \\
\hline $0.5-1$ hour compared to 0.5 hours & $0.014(0.052,0.081)$ & $0.012(0.056,0.079)$ & $0.014(0.054,0.081)$ \\
\hline 1 hour compared to 0.5 hours & $0.035(0.115,0.045)$ & $0.036(0.142,0.070)$ & $0.038(0.147,0.071)$ \\
\hline Trend $p$ & 0.860 & 0.658 & 0.663 \\
\hline
\end{tabular}

Note: Boldface indicates significance. Model 1 was adjusted for age and gender. Model 2 was further adjusted for mother's education and BMI; father's education and BMI; perception of crime in local area; average hours of sleep per night; birth weight; duration of breastfeeding; bad diet score (based on consumption of sodas, iced tea, cakes, chocolate, hamburgers, and pizza); and number of fruit portions eaten per week. Model 3 was further adjusted for time spent in physical activity per week. Coefficients are generalized linear model coefficients; coefficients indicate mean differences (in adiposity markers) between the reference category ( 0.5 hours a day) and each of the other PC frequency groups (e.g., a value of 0.06 indicates that a specific category had a mean score that is 0.06 units higher than that of the referent group).

aiastolic and systolic blood pressure are logged variables (base 10). Coefficients presented here have been back-transformed to their natural scale by raising 10 to the power of the logged coefficient and deducting 1.

$\mathrm{PC}$, personal computer

did find associations for some cardiovascular markers (HOMA-R score [homeostasis model assessment insulin resistance score] and C-peptide levels) after controlling for weight group, fat and sugar intake, and physical activity, but not for total cholesterol, low-density lipoprotein cholesterol, HDL cholesterol, triglycerides, or glycated hemoglobin. ${ }^{5}$

The lack of an association between screen time and RHR was not surprising given that intensities in the higher end of the physical activity spectrum mainly are associated with cardiorespiratory fitness, ${ }^{20}$ for which RHR is an indicator. It is unclear whether the observed associations between TV viewing and blood pressure and overall cardiovascular risk score were due to the sitting that TV viewing entails, or to other, residual causes. The lack of association with other forms of screen time (PC, games) that also typically are performed while sitting suggest that $\mathrm{TV}$ viewing also might reflect other behavioral risk factors, such as consuming high-energy snack foods and/or the influence of TV advertisements on unhealthy behaviors. ${ }^{9}$ 
This explanation is supported by two studies of children aged 3-8 years ${ }^{12}$ and adolescents aged 13-17 years ${ }^{13}$ that found that total (objectively assessed [i.e., non-type specific]) sedentary behavior was not associated independently with cardiovascular risk indicators, whereas self-reported TV viewing was.

In the present analyses, it was only possible to adjust for simple measurements of diet (number of pieces of fruit eaten per week and a crude frequency-based index of unhealthy foods consumption); hence, residual behavioral confounding might remain. The associations between TV and overall cardiovascular risk reported here may be mediated partly by adiposity, as recent results show that TV viewing is associated with BMI and sum of skinfolds in the full PPSOC sample (unpublished observations, 2012). Prospective evidence shows that higher adiposity predisposes people to higher levels of sedentary behavior in general, and higher TV viewing might be a potential extension of this finding. ${ }^{21}$ Also, TV viewing is associated with psychological distress in children. ${ }^{22}$ Given that psycholog- 
ical factors are linked to the early pathogenesis of cardiovascular disease, ${ }^{23,24}$ this may be another potential explanation of the associations observed in the present study.

An alternative explanation of the differential associations between types of screen time and the cardiovas-

cular risk indicators is differential measurement error of each screen time indicator and behavior. That is, TV might be recalled and observed more accurately by the parents than games and PC use because, for example, viewing of specific programs aid recall and accuracy. Although no information could be located that specifically deals with parental proxy measures, TV-viewing measurement has shown the highest validity among all sedentary behavior measures in adults. ${ }^{1}$ Although screen-time measures in children in general have acceptable test-retest reliability, their validity remains unknown, mainly because of difficulties in measuring them.

\section{Strengths and Limitations}

The strengths of the current study are the large population sample and the availability of data on multiple screen-related behaviors and cardiovascular risk markers. A major limitation of this study is the crosssectional design, which precludes causal inferences. Other limitations include the absence of blood variables, such as lipids and indicators of glucose metabolism that would allow more-comprehensive examination of the associations of the exposures with cardiovascular risk.

Clinical definitions of cardiovascular risk in children are not developed, and thus, the results should not be interpreted as identifying children with higher levels of clinical risk according to their screen time. Screen time and physical activity reports were obtained by parental proxy, which might be inaccurate, as it relies on accurate recall, and parents are not always with their children. This is perhaps a more serious issue for physical activity that can be performed outside the domestic environment and less so for screen time that mostly is performed at home. Previous studies have shown that parental proxy questionnaires of children's physical activity have poor to fair agreement with accelerometer-based estimates. ${ }^{11}$ Nevertheless, the results of the models show that the relevant measuring methodologies had a good degree of convergent validity. Finally, as the screen time data were catego-

\section{Conclusion}

The current study found that TV viewing, but not PC time or game time, is associated with cardiovascular risk. Future studies should investigate the role of TV viewing in cardiovascular health among youth, using prospective designs.

rized into groups, it is possible that small associations were not detected, so the associations reported here are conservative estimates. 
This study was supported by a grant of the Fundação para a Ciência e Tecnologia FCOMP-01-0124-FEDER-007483. This paper is a part of the research arising from Career Development Fellowships (ES and RJ) supported by the National Institute for Health Research (NIHR). The views expressed in this publication are those of the authors and not necessarily those of the Nutrition and Health Sciences Department, NIHR, or the Department of Health.

No financial disclosures were reported by the authors of this paper.

\section{References}

1. Janssen I, LeBlanc AG. Systematic review of the health benefits of physical activity and fitness in school-aged children and youth. Int J Behav Nutr Phys Act 2010;7(40).

2. Andersen LB, Harro M, Sardinha LB, et al. Physical activity and clustered cardiovascular risk in children: a cross-sectional study (The European Youth Heart Study). Lancet 2006;368(9532):299-304.

3. Ekelund U, Brage S, Froberg K, et al. TV viewing and physical activity are independently associated with metabolic risk in children: the $\mathrm{Eu}-$ ropean Youth Heart Study. PLoS Med 2006;3(12):e488.

4. Martinez-Gomez D, Eisenmann JC, Healy GN, et al. Sedentary behaviors and emerging cardiometabolic biomarkers in adolescents. J Pediatr 2011;160(1):104-10.

5. Kang HT, Lee HR, Shin JY, Shin YH, Park BJ, Lee YJ. Association between screen time and metabolic syndrome in children and adolescents in Korea: the 2005 Korean National Health and Nutrition Examination Survey. Diabetes Res Clin Prac 2010;89:72-8.

6. American Academy of Pediatrics CoPE. Children, adolescents, and television. Pediatr 2001;107:423-6.

7. Tremblay MS, LeBlanc AG, Janssen I, et al. Canadian sedentary behavior guidelines for children and youth. Appl Physiol Nutr Metab 2011;36:59-64.

8. Department of Health. Physical activity, health improvement and protection. Start active, Stay active: a report on physical activity for health from the four home counties. London: Chief Medical Officers, 2011.

9. Australian Government Department of Health and Ageing. Physical activity recommendations for 5-12 year olds. Canberra, Australia: Department of Health and Ageing, 2004.

10. Carlson V, Janssen I. Volume, patterns, and types of sedentary behavior and cardio-metabolic health in children and adolescents: a crosssectional study. BMC Public Health 2011;11:274.

11. Stamatakis E, Hamer M, Tilling K, Lawlor D. Sedentary time in relation to cardio-metabolic risk factors: differential associations for self-report vs accelerometry in working age adults. Int J Epidemiol 2012;41: 1328-37.

12. Cleland VJ, Schmidt MD, Dwyer T, Venn AJ. Television viewing and abdominal obesity in young adults: is the association mediated by food and beverage consumption during viewing time or reduced leisuretime physical activity? Am J Clin Nutr 2008;87:1148-55. 
13. Martinez-Gomez D, Tucker J, Heelan KA, Welk GJ, Eisenmann JC. Associations between sedentary behavior and blood pressure in young children. Arch Pediatr Adolesc Med 2009;163(8):724-30.

14. Pardee PE, Norman GJ, Lustig RH, Preud'homme D, Schwimmer JB. Television viewing and hypertension on obese children. Am J Prev Med 2007;33(6):439-43.

15. Tremblay MS, LeBlanc AG, Kho ME, et al. Systematic review of sedentary behavior and health indicators in school-aged children and youth. Int J Behav Nutr Phys Act 2011;8:98.

16. Jago R, Stamatakis E, Gama A, et al. Parent and child screen-viewing time and home media environment. Am JPrev Med 2012;43(2):150-8.

17. Lubans DR, Hesketh K, Cliff DP, et al. A systematic review of the validity and reliability of sedentary behaviour measures used with children and adolescents. Obes Rev 2011;12(10):781-99.

18. Wijndaele K, Healy GN, Dunstan DW, et al. Increased cardiometabolic risk is associated with increased TV viewing time. Med Sci Sports Exerc 2010;42(8):1511-8.

19. Clark BK, Sugiyama T, Healy GN, Salmon J, Dunstan DW, Owen N. Validity and reliability of measures of television viewing time and other non-occupational sedentary behaviour of adults: a review. Obes Rev 2009;10(1):7-16.

20. Aires L, Silva P, Silva G, Santos M, Ribeiro JC, Mota J. Intensity of physical activity, cardiorespiratory fitness, and body mass index in youth. J Phys Ac Health 2010;7(1):54-9.
21. Ekelund U, Luan J, Sherar LB, Esliger DW, Griew P, Cooper A; International Children's Accelerometry Database (ICAD) Collaborators. Moderate to vigorous physical activity and sedentary time and cardiometabolic risk factors in children and adolescents. JAMA 2012; 307(7):704-12.

22. Hamer M, Stamatakis E, Mishra G. Psychological distress, television viewing, and physical activity in children aged 4 to 12 years. Pediatrics 2009;123(5):1263-8.

23. Roemmich JN, Feda DM, Seelbinder AM, Lambiase MJ, Kala GK, Dorn J. Stress-induced cardiovascular reactivity and atherogenesis in adolescents. Atherosclerosis 2011;215(2):465-70.

24. Roemmich JN, Lobarinas CL, Joseph PN, Lambiase MJ, Archer Iii FD, Dorn J. Cardiovascular reactivity to psychological stress and carotid intima-media thickness in children. Psychophysiology 2009;46(2): 293-9.

\section{Appendix}

\section{Supplementary data}

Supplementary data associated with this article can be found, in the online version, at http://dx.doi.org/10.1016/j.amepre.2013.01.020. 